\title{
Lexis
}

Journal in English Lexicology

Book reviews | 2019

\section{Sandrine SORLIN, La stylistique anglaise. Théories et} pratiques.

Presses universitaires de Rennes, 2014, 228 pages

\section{Adam Wilson}

\section{OpenEdition}

\section{Journals}

Édition électronique

URL : http://journals.openedition.org/lexis/3479

DOI : $10.4000 /$ lexis.3479

ISSN : 1951-6215

Éditeur

Université Jean Moulin - Lyon 3

Référence électronique

Adam Wilson, "Sandrine soruin, La stylistique anglaise. Théories et pratiques. », Lexis [En ligne], Recensions, mis en ligne le 24 mars 2019, consulté le 24 septembre 2020. URL : http:// journals.openedition.org/lexis/3479; DOI : https://doi.org/10.4000/lexis.3479

Ce document a été généré automatiquement le 24 septembre 2020.

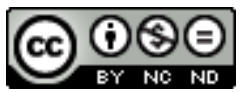

Lexis is licensed under a Creative Commons Attribution-NonCommercial-NoDerivatives 4.0 International License. 


\title{
Sandrine SORLIN, La stylistique anglaise. Théories et pratiques.
}

Presses universitaires de Rennes, 2014, 228 pages

\author{
Adam Wilson
}

\section{RÉFÉRENCE}

Sandrine Sorlin

La stylistique anglaise. Théories et pratiques. Presses universitaires de Rennes, Didact

Anglais, Rennes, 2014. ISBN : 978-2-7535-3446-9, Prix : 16€, 228 pages

1 La stylistique anglaise. Théories et pratiques est un manuel qui vise à offrir une introduction aux théories, concepts, méthodes et pratiques de la stylistique anglaise. Écrit par Sandrine Sorlin, alors Professeure de linguistique anglaise à Aix-Marseille Université et auteure d'un large éventail de travaux en linguistique, cet ouvrage a pour but de guider les lecteurs dans l'étude de la langue anglaise d'un point de vue stylistique, dans les analyses de textes et dans l'exploration de pratiques stylistiques. Il est composé de cinq chapitres principaux ainsi que d'une introduction, une conclusion et une préface écrite par Manuel Jobert.

2 La courte préface de M. Jobert, jadis président de la Société de Stylistique Anglaise (SSA), souligne les nombreux points forts de l'ouvrage de S. Sorlin, dont certains seront repris dans cette recension. Il insiste particulièrement sur le fait que $\mathrm{S}$. Sorlin réussit à présenter l'immensité du champ de la stylistique, abordant de multiples concepts et méthodes provenant de courants différents, à la fois anglophones et francophones, tout en étant concis et cohérent. M. Jobert positionne le travail de S. Sorlin comme étant déjà un ouvrage de référence dans le champ de la stylistique.

Suite à la préface de M. Jobert, S. Sorlin offre une introduction à son ouvrage dans laquelle elle rappelle l'histoire de la stylistique en tant que champ d'étude, avant d'en proposer sa définition. L'auteure insiste sur le caractère scientifique de la stylistique et la situe au carrefour de la linguistique et des études littéraires avant d'expliquer que : 
« la stylistique vise à saisir la façon dont un discours (écrit ou oral) utilise les potentialités de la langue à des fins spécifiques dans un contexte particulier de production et de réception $»($ p. 12)

Cette définition constitue le point de départ du «triple objectif » annoncé par S. Sorlin pour son ouvrage: une introduction à l'étude de la langue anglaise, aux analyses de textes et aux pratiques stylistiques. S. Sorlin conclut son introduction par un bref rappel de l'histoire de la stylistique, ce qui constitue également la structure fondamentale des cinq chapitres principaux de son ouvrage.

5 Le premier chapitre, intitulé « Du micro-linguistique au macro-contexte (et retour)» (p. 21-51), est consacré à l'étude de la grammaire anglaise et son utilisation pour différents effets stylistiques. Pour ce faire, S. Sorlin se focalise tout d'abord sur l'utilisation des «marqueurs " grammaticaux dans des textes littéraires et leurs rôles dans l'élaboration des relations entre le narrateur, les personnages et le lecteur ainsi que dans la construction de l'expérience humaine et des représentations fictives du monde. Ensuite, l'auteure aborde les facteurs linguistiques et non linguistiques des concepts de genre, de registre et de style, définissant ces termes et expliquant leurs distinctions. S. Sorlin conclut ce chapitre avec une proposition pour une «stylistique socio-cognitive (inclusive)» (p.46), rassemblant tous les éléments étudiés dans différents courants de la stylistique de nos jours et permettant à l'auteure de dresser un état de l'art de la stylistique historique et actuelle.

Ce premier chapitre introduit le lecteur à deux points forts de l'ouvrage de S. Sorlin qui sont omniprésents dans les autres chapitres du livre. Premièrement, S. Sorlin propose une introduction conséquente et variée aux théories et aux concepts non seulement de la stylistique, mais aussi de la linguistique, s'appuyant sur de solides synthèses des travaux d'auteurs majeurs dans ces deux vastes champs (et notamment des œuvres de Paul Simpson, M.A.K. Halliday et Douglas Biber dans ce chapitre). Deuxièmement, ce considérable travail théorique est complémenté par l'utilisation systématique d'exemples et de contre-exemples, afin d'illustrer de façon très pédagogue les concepts présentés. Cette approche permet une expérience de lecture cohérente et facile, malgré le niveau élevé de détail dans les explications.

7 Le chapitre 2, «Grammaire et style » (p. 53-96), approfondit ce travail sur l'interaction entre les différentes formes grammaticales de la langue anglaise et les effets stylistiques. Le chapitre est divisé en deux parties qui visent à « montrer comment on peut apprendre la grammaire anglaise dans une perspective stylistique » (p. 53) et ainsi comprendre l'utilisation de certains marqueurs et les effets que ceux-ci peuvent avoir. Dans la première partie, S. Sorlin aborde les marqueurs de style : prenant appui sur les travaux de Geoffrey Leech et Mick Short, elle explique qu'il s'agit en premier lieu d'apprendre à identifier et à catégoriser les marqueurs lexicaux et syntaxiques d'un texte. Par la suite, l'auteure traite de la cohésion et de la gestion de l'information et montre, en s'appuyant sur la linguistique hallidayienne, les différentes façons dont l'information peut être structurée et les effets stylistiques de ces partis pris. Ce dernier élément est illustré par une mise en pratique à travers l'analyse d'un long extrait d'un roman de Mark Haddon, éclairée par des contre-exemples tirés d'un livre de Ian McEwan. La deuxième partie du chapitre 2 aborde les concepts de "défamiliarisation » et de «foregrounding » et $\mathrm{S}$. Sorlin montre comment certains procédés linguistiques, tels que l'utilisation de certaines collocations, l'emploi de néologismes ou des jeux avec les 
régularités et irrégularités linguistiques peuvent créer des effets de surprise ou aider l'auteur à mettre en relief certains éléments de la narration.

8 Ce deuxième chapitre révèle ainsi deux autres atouts de cet ouvrage. Premièrement, S. Sorlin emploie une grande diversité de sources : non seulement en ce qui concerne les œuvres primaires mais également au niveau des sources secondaires. Cette ouverture d'esprit et cette capacité à puiser dans une vaste culture linguistique et littéraire ouvrent énormément de pistes en termes de lectures possibles. Deuxièmement, le chapitre 2 contient les premiers «assignments» du livre. Ces suggestions d'activités d'écriture qui permettent au lecteur d'appliquer les éléments traités dans l'ouvrage deviennent un élément fondamental des chapitres suivants et contribuent au caractère innovant et pédagogique de l'ouvrage.

Le troisième chapitre, «Cadre narratif et focalisation » (p. 97-127), aborde la littérature en tant qu'acte de communication. S. Sorlin explique comment cette communication peut avoir lieu malgré l'absence de contact direct entre l'auteur et le lecteur d'un texte, et montre que la plupart des approches de la stylistique tendent à « effacer » l'un de ces acteurs. En réponse, S. Sorlin présente la théorie pragmatique de l'interprétation de Jean-Jacques Lecercle qui place le texte au centre du processus d'interprétation. Cette théorie sert de point de départ pour un exposé, soutenu systématiquement par des exemples tirés d'une grande variété de textes littéraires, des différents positionnements du narrateur dans un texte et des effets que ceux-ci peuvent avoir sur l'interprétation de ce dernier. Ensuite, S. Sorlin aborde les catégories narratologiques, s'appuyant tout d'abord sur les travaux de Gérard Genette pour montrer comment les textes peuvent être catégorisés selon le degré de "participation" du narrateur à l'action du récit. L'auteure synthétise les recherches de G. Genette et de P. Simpson afin de présenter les caractéristiques linguistiques des "phénomènes de focalisation " (p. 109), permettant de distinguer le niveau de connaissances du narrateur sur les personnages et sur l'action d'un récit. Le chapitre se termine par une discussion concernant les effets pragmatiques de l'utilisation de pronoms « inhabituels » tels que we, you ou they. Tout comme le chapitre précédent, le chapitre 3 est ponctué d' assignments et d'exemples illustrés qui aident le lecteur à mettre en pratique les éléments théoriques abordés.

Le chapitre 4 s'intitule « La (re)présentation des paroles et des pensées » (p. 129-169) et aborde les différentes façons de présenter ce que les personnages dans un récit pensent ou disent. Tout d'abord, S. Sorlin rappelle la complexité de la notion de paroles ou pensées "rapportées", étant donné qu'il s'agit d'éléments fictifs, et ainsi jamais énoncés. Par la suite, l'auteure se fonde à nouveau sur les travaux de G. Leech et M. Short afin de présenter les catégories de représentation des paroles et des pensées sous forme de continuum. Elle saisit l'occasion de peaufiner ces catégories avant de montrer comment elles peuvent être adaptées également à des actes d'écriture. Elle propose ensuite des sous-catégories transversales pour décrire la présentation de paroles, pensées et actes d'écriture. En se fondant sur les travaux de Monique de Mattia-Viviès, S. Sorlin identifie le Discours Indirect Libre comme un cas particulièrement intéressant et consacre une partie du chapitre à l'analyse de ce phénomène, illustrée par des études de cas. S. Sorlin aborde ensuite les critères linguistiques qui permettent de différencier le monologue intérieur du stream of consciousness avant de se focaliser sur les échanges verbaux entre les personnages d'un récit, et sur le rôle de ces échanges dans le développement des traits de caractère. Cette 
dernière partie constitue une véritable introduction à la pragmatique - abordant entre autres la présupposition, les actes de langage, la politesse et le concept de face, les apports de Grice (coopération, maximes et implicature) et Sperber et Wilson (le principe de pertinence) - qui a le mérite d'appliquer ce champ à un domaine très peu étudié sous cet angle : le théâtre.

11 Le chapitre 5, «Le tournant cognitif en stylistique » (p. 171-202), constitue un apport considérable de l'ouvrage de S. Sorlin. L'auteure initie le lecteur à ce courant récent en stylistique, qui est caractérisé par sa complexité mais présenté ici de façon très concise et accessible. Il s'agit également d'une occasion pour S. Sorlin de présenter de nombreux travaux anglophones à un public francophone et elle propose systématiquement des traductions françaises des notions présentées. Le chapitre est organisé en quatre parties. Tout d'abord, S. Sorlin aborde les liens entre la cognition et la lecture, et surtout l'importance de l'activité du lecteur dans la constitution d'un texte, ce qui permet une remise en question de certaines notions stylistiques présentées dans des chapitres précédents. Dans un second temps, S. Sorlin présente la théorie des mondes textuels de Paul Werth et Elena Semino et montre comment les mondes de la fiction sont élaborés linguistiquement et abordés par le lecteur. Ensuite, l'auteure applique à l'acte de lecture plusieurs théories issues des études sur la cognition et notre perception du monde. Pour terminer, les notions de "métaphore " et de « métonymie » sont présentées d'un point de vue cognitif et linguistique.

12 S. Sorlin clôture son ouvrage par une courte conclusion qui rappelle au lecteur le caractère interdisciplinaire de la recherche en stylistique, avant de présenter brièvement de nombreuses "sous-disciplines» des études stylistiques. L'auteure détaille rapidement le déroulement méthodologique d'une étude stylistique en se focalisant sur les voies d'entrée possibles pour un projet de recherche, le choix de corpus et les différentes approches analytiques.

13 La fin de la conclusion vient souligner deux qualités majeures de l'œuvre présentée ici. Premièrement, S. Sorlin résume: "Loin d'être exhaustifs, les cinq chapitres de cet ouvrage ont offert un panorama des différentes théories exploitées en stylistique anglaise» (p. 207). Si l'auteure nous confesse à travers cette phrase son objectif principal, force est de constater qu'il a été largement atteint. En effet, le vaste panorama de la stylistique qui nous y est proposé est sans égal, et $\mathrm{S}$. Sorlin va encore plus loin en proposant régulièrement des exemples illustratifs, des mises en pratique et des activités d'application. Deuxièmement, S. Sorlin termine en louant l'ouverture d'esprit permise par l'étude de la stylistique, que ce soit en termes de types de textes étudiés, de méthodes, d'objets ou de questions d'analyse. Ce point de vue nous révèle l'ouverture dont fait preuve l'auteure elle-même, présentant dans son ouvrage un large éventail de théories et de pratiques stylistiques, sans faire preuve de dogmatisme ou de favoritisme vis-à-vis des domaines abordés.

14 La stylistique anglaise. Théories et pratiques constitue un livre incontournable pour tout étudiant, enseignant ou chercheur qui s'intéresse à la stylistique, à la langue anglaise ou à la linguistique de façon plus générale. Le «triple objectif» du livre annoncé par S. Sorlin dans son introduction - "étude de la langue, analyse de textes et pratique stylistique» (p.19) - est atteint avec brio. L'ouvrage se distingue par son fond théorique considérable, marié à une approche hautement pédagogique, le tout appuyé sur une grande diversité de sources primaires et secondaires et une ouverture scientifique remarquable. 
15 Face à de tels atouts, il est difficile de signaler de réelles failles à cet ouvrage, sinon qu'il a les défauts de ses qualités. Ainsi, la lecture est parfois rendue difficile par la richesse théorique et la densité des concepts abordés. À de rares occasions, il semble ardu de suivre le fil conducteur du texte. D'autre part, cette diversité d'apports peut laisser penser que certains concepts ou questions auraient pu être développés davantage, laissant le lecteur sur sa faim. En revanche, l'appétit suscité peut aussi être envisagé comme un point de départ idéal pour des lectures ou recherches futures.

En conclusion, comme le suggère Manuel Jobert dans sa préface, La stylistique anglaise. Théories et pratiques est un ouvrage qui fera date dans la discipline de la stylistique. Ce livre détaillé mais accessible va au-delà d'une simple introduction à la stylistique. S. Sorlin offre un ouvrage de référence pour les domaines de la stylistique et de la linguistique anglaise mais également un travail qui pousse le lecteur à se poser des questions fondamentales sur les rapports entre le langage et ses fonctions pour les êtres humains.

\section{AUTEURS}

\section{ADAM WILSON}

Adam Wilson, Université de Lorraine, France.

Adam Wilson est actuellement Maître de conférences en Linguistique dans le Département LEA de l'Université de Lorraine à Metz, où il est affilié à l'équipe IDEA (EA 2338). Ses recherches portent sur les influences réciproques entre le langage - dans ses formes et usages - et les phénomènes sociaux, et ce dans le cadre des « nouveaux » contextes sociolinguistiques liés à la globalisation. 